Kalpa Publications in Engineering
Volume 3, 2020, Pages 182-186
Proceedings of International Sym-
posium on Applied Science 2019

\title{
Coverage Motion Planning Using Graph In Forest Environment In Field Robot
}

\author{
Ayumu Tominaga ${ }^{1}$, Ryusuke Fujisawa $^{1}$, Eiji Hayashi $^{1}$ \\ ${ }^{1}$ Kyushu Institute of Technology, Japan \\ tominaga@mmcs.mse.kyutech.ac.jp
}

\begin{abstract}
This paper addresses the problem of using a mobile, autonomous robot to manage a forest whose trees are destined for eventual harvesting. We have been fourcussing a eliminate weeding operation because it is one of the hard work in the forestry works. This research proposing the computation of trajectory capable of traversing in entire forest. The method is based on graph whose vertices are trees located in forest. Trees in located in the forest will be treated as vertices in a graph. In the first, the initial graph is made with considering the safety for the robot. Next, editting the initial graph to be Eulerian, and finally, the Hamiltonian circuit is obtained which could be used for trajectory. By our proposed method, the trajectory which feasible route for traversing of entire of forest would be obtained. In the experiment, we show the result of the method applying to actual artificial forest.
\end{abstract}

\section{Introduction}

This research addresses the application of an autonomous robot to forestry. Forestry is the industrial that cultivates trees and uses a forest for economically. However, employeers are decreasing year by year, insufficient the work force. As the result an unmanaged forest area have been increasing and to utilize effectively the forest resource has not been done. On the other hand, for application of forest resource management is well established [1]. On the other hand, Shirai and et al. has suggested that modenization and mechanization of forestry should be more developed from view of safety and productivety by inplementation of the autnomous mobile robot and robotics technology [2].

In this research, we have been focussing a weeding operation in forest iudustry work, and proposing its labor-saving by implementation of the autonomous robot. In such task, the navigation of the robot is challenging part. To avoid a obstacle or trees is nessesary function for the system, in addition, to plan the route for increase of effective of automatic weeding is important things. This paper discribes a trajectory computation in actual artificial forest. The proposing method is based on graph modeling of the forest with treating trees as vertices in a graph. Obtained graph could be used as the feasible 
trajectory for terversing entire of forest. We present the procedure of proposed trajectory computation, and finally, show the result which applied to actual artificial forest.

\section{Trajectory Genration Based On Grpaph}

\subsection{Graph Modeling of Forest}

In this research, an artificial forest constructed by trees is modeled based on a graph structure in order to generate the trajectory for traversing entire forest. A graph is data structure represented by a set of vertices and a set of edges [3]. Trees constructing the forest can be represented by a graph $G=$ $(V, E)$, where $V$ is a set of vertices, $E$ is a set of edges. A vertex $v \in V$ represents an individual tree, and an edge $e \in E$ joining vertices $v S$ and $v t$ represents a line between these trees. In this research, the position of tree $(x i, y i)$ gives to the vertex corresponds of its, therefore the edge will represent the segment in two dimentional Euclidian space. By applying such graph modeling to the artificial forest, given graph could be drawn on 2D map, in addition, any algorithms in graph theory can be utilized for the forestry robot.

To navigate the robot in actual environment, we have been focussed a line graph in graph theory [4]. The line graph is the graph derived from the original graph, defined as below. In this paper, the line graph is denoted as $L(G)$.

- $L(G)$ has a set of vertices whose vertex corresponding a edge in $G$.

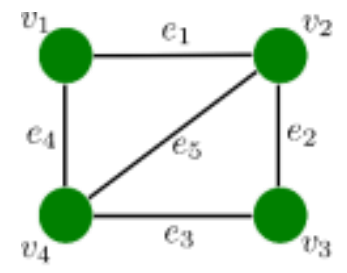

$G$



$L(G)$

Figure 1: Example of the line graph

- $\quad$ Two vertices $v s, v t \in V(L(G))$ are adjacent if the corresponding edges in $G$ are incident.

The exapmle of the line graph is shown in Figure 1. As shown this example, $L(G)$ is the graph with edges in $G$ replaced to by vertices and represents the condition of edge joining of the original graph. We impose a natural geometry on the line graph, taking the position of the vertices of $L(G)$ to be the mid points of the edges of $G$.

\subsection{Procedure of Trajectory Generation}

If the original graph $G$ is Eulerian graph, then its line graph will be Hamiltonian graph [4]. Eulerian graph is the graph containing the path which capable of traversing all edges with using each edge exactly once. And Hamiltonian graph is the graph having the path which capable of visiting all vertices with using each vertex exactlly once. This charactaristic of the line graph is obvious by line graph definitions. By proposed graph modeling way of the artificial forest, $L(G)$ 


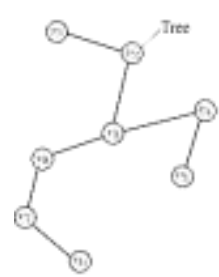

(a) Initial graph

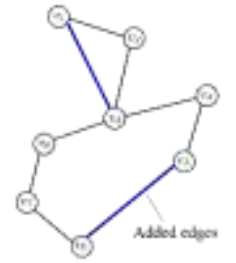

(b) Fulerisn graph

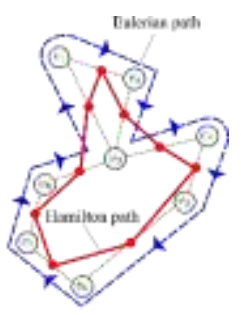

(c) Firal irajectury

Figure 2: The procedure of global trajectory generation

will be the graph whose edges join midpoints of any two trees. Therefore, if we able to make Eulerian graph using tree locations, it is considered that the Hamiltonian path capable of visiting all midpoints will be obtained. Such trajectory fiasible of visiting tree's midpoints in the forest is obtained by the procedure as mention below.

1. Making initial graph $G$ (Figure.2 (a)).

2. Editing $G$ to be Eulerian (Figure.2 (b)).

3. Computing the Hamiltonian path (Figure.2 (c)).

Each process are mention below.

\subsubsection{Make Initial Graph}

In this paper, the graph $\mathrm{G}$ constructed using trees as the set of vertices is called initial graph. In this process, each edge have to connect with considering safty of neighborhood of its because the robot will be navigated as passing through the midpoints of the edge. As the simple idea, if the situation of two trees is the line-of-sight then these will be connected by the edge. For the aritificial forest composed several trees, it would be hard work that the pair of trees exist on the line-of-sight each others. Thus, the system determines whether tree pairs is on the line-of-sight or not as shown in Figure.3. Here $v s$ and $v t$ represent a pair of trees, $v r$ is the others. If the distance $d r$ between $v r$ and the segment consisted by $v s$ and $v t$ is less than a threashold value $\overline{d r}$ then this segment will add to the set of edge in the initial graph $G$. Additionally, the length of edge have to be longer than the robot size.

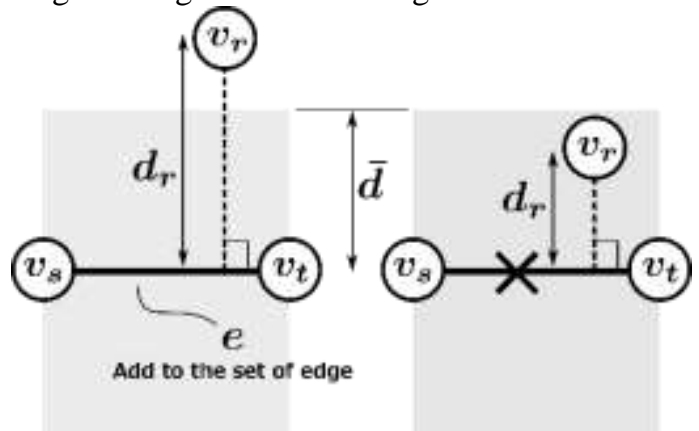

Figure 3: Line-of-Sight condistion of tree pairs

\subsubsection{Editing $\boldsymbol{G}$ to be Eulerian}

Necessary and sufficient condition of a graph to be Eulerian is the all vertices in $G$ is having even number of edges (参考文献). The Eulerian graph would be obtained by the procedure as mention below.

1. Extraction vertices having odd number degree in $G$. The set of these vertices is denoted as $V o$. 
2. Computing the minimum cost matching $M$ in the complete graph $K O=(V o, E o)$.

3. Addition edges in $M$ to the original graph $G$. In finally, Eularian graph $G *$ would be obtained.

A maching in graph theory is extraction a subset of edges whose edge have not share the common vertex in a graph. Especially, minimum cost matching is to find the subset of edges that the total cost will be minimum. To find such matching, the 1-0 Integer Linear Programing method was used in the system. Finding the matching $M$ using odd degree vertices, and adding $M$ to the original graph, it can be edit that every vertices in $G$ to be even degree vertices. Therefore, Eulerian graph $G *$ would be obtained.

\subsubsection{Computing Hamiltonian Path}

The Eulerian path in $G *$ that can pass through every edge with using each edge exactrlly once will be found using Depth First Search (DFS) method. As the results, the ordered set of edges would be obtained. It can be expressed as shown equation 1. Finally, by calculating the midpoints of each edge of the set, the trajectory expressed by the set of vertices (these are midpoints of tree pairs) would be acquired, as shown in equation 2 and 3.

\begin{tabular}{|c|c|}
\hline $\boldsymbol{B}=\left\{e_{1}, e_{2}, \ldots, e_{j}\right\}$ & $(1)$ \\
\hline $\boldsymbol{P}=\left\{\boldsymbol{p}\left(e_{1}\right), \boldsymbol{p}\left(e_{2}\right), \ldots, \boldsymbol{p}\left(e_{j}\right)\right\}$ & $(2)$ \\
\hline $\boldsymbol{p}\left(e_{j}\right)=\left\{\frac{x}{2}-x_{t}, y_{s}+y_{t}\right\}$ & \\
2 & $(3)$ \\
\hline
\end{tabular}

\section{Experiment Of Trajectory Generation In Real Forest}

The trajectory computation method described in the previous section was applied to an actual artificial forest, and the obtained trajectory is evaluated. The figure 4 shows two artificial forest data that we used in this experiment. Both distribution of tree location are different each other. The number of trees in each forest are almost same, "Forest A" has 38, "Forest B" has 32. Tree locations of Forest A was measured by RTK-GPS. Tree locations of Forest B was measured by Terrestrial-Laser Scanner (TLS) instruments. The result of trajectory generation shown in Figure 5 whose the threshold parameter of line-of-sight condition $\overline{d r}$ and the threshold of minimum distance between tree pair $\overline{d e}$ were 1.0 and 2.0 respectively. As this figures, Both trajectorys has generated as traversing the entire forest. In addition, the locally high planting density region such as somwhere in Forest B had avoided. Therefore, the proposed line graph based method could be compute for the actual artificial forest and tree locations. However, the generated trajectory has not generated in a lot of free space in actual forest, so it cannot be said that complete coverage could be achieved. 


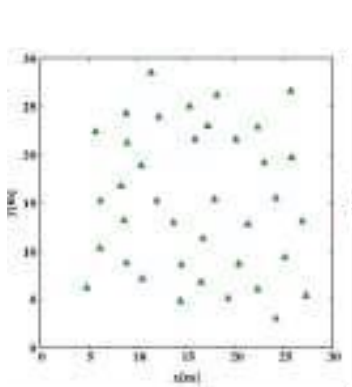

Foerst A

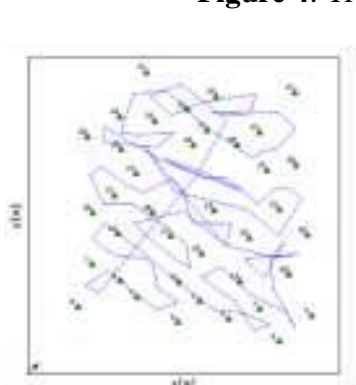

Foerst A

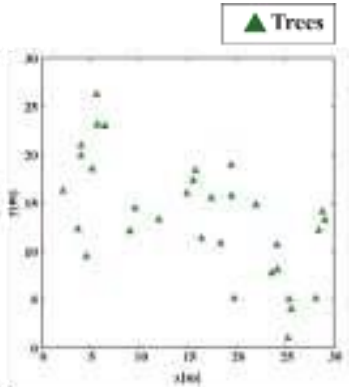

Foerst B

ree locations

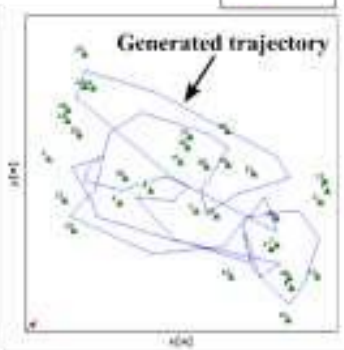

Foerst B

Figure 5: Result of trajectory generation of both forest

\section{Conclusions}

This paper has proposed a trajectory generation method based on graph structure and line graph. In the method, trees are treated as vertices in the grpah. And using the characteristics of line graph as the background, we could get the Hamiltonian curcuit capable traversing of entir forest. However, increasing the effectiveness of automatic weeding by the mobile robot would be achieved by considering the distribution of trees and evaluation of relation between generated trajectory and it.

\section{References}

R. Parker, K. Bayne, and P. W. Clinton, ”Robotics in Forestry,” New Zealand J. Of Forestry, Vol. 60, pp. 8-14, 2016.

Y. Shirai, and et. al., "Hopes for Robotics Advancing Forest Industry,” Vol. 28, No. 1, pp. 43-48, 2010.

F. Harary, "Graph Theory," Reading MA, Addison-Wesley, 1969.

F. Fujie and P. Zhang, "Line Graphs and Power of Graphs," in Convering Walks in Graphs, Springer, 2014 